Steuer, Dunja; Grießbach, Gert:

Bildfolgensegmentierung auf der Basis komplexer adaptiver Schätzverfahren

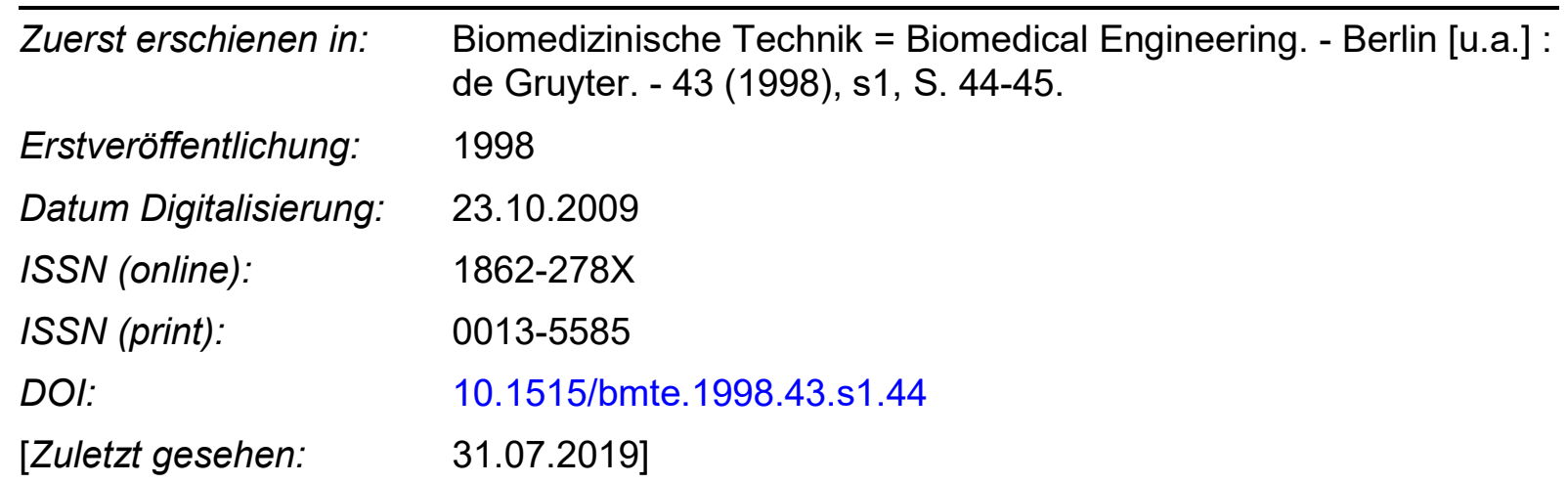

„Im Rahmen der hochschulweiten Open-Access-Strategie für die Zweitveröffentlichung identifiziert durch die Universitätsbibliothek IImenau."

"Within the academic Open Access Strategy identified for deposition by IImenau University Library."

„Dieser Beitrag ist mit Zustimmung des Rechteinhabers aufgrund einer (DFGgeförderten) Allianz- bzw. Nationallizenz frei zugänglich."

"This publication is with permission of the rights owner freely accessible due to an Alliance licence and a national licence (funded by the DFG, German Research Foundation) respectively."

\section{DFG}

Nationallizenzen 


\title{
Bildfolgensegmentierung auf der Basis komplexer adaptiver Schätzverfahren
}

\author{
Steuer D. Grießbach G. \\ Technische Universität IImenau, Institut fur Biomedizinische Technik und Informatik, D-98684 IImenau
}

\section{EINLEITUNG}

Wie auch in der Biosignalanalyse spielt es in der Bildverarbeitung eine große Rolle, Veränderungen im Signalverlauf bzw. im Bildfolgenverlauf zu detektieren. In der Biosignalverarbeitung haben sich adaptive rekursive Schätzverfahren in vielen Applikationen unter Anwendung einer Vielzahl von Parametern im Zeit- und Frequenzbereich bewährt. Ausgehend von diesen Erfahrungen wird ein Lösungsansatz vorgestellt, der es gestattet, diese Algorithmen auf die komplexeren Problemstellungen der Bildverarbeitung zu transformieren. Ziel der Untersuchungen ist es, mit einem vertretbaren Aufwand Phasen in einer Bildsequenz zu erkennen, in denen keine oder nur eine geringfügige Bildänderung stattfinden, und die Sequenz so in bestimmte Abschnitte zu segmentieren. An einem konkreten Beispiel aus einem von uns bearbeiteten DFG-Projekt zur Kognitionsforschung werden die vorgestellten Verfahren zur Segmentierung von EEGParameter-Mapsequenzen benutzt. Dabei kommt es darauf an, aus den Mapsequenzen Abschnitte zu detektieren, die mit einer Aktivitätsverschiebung der Informationsverarbeitung im Gehim einhergehen. In Verbindung mit anderen Verfahren zur Signalsegmentierung erhofft man sich dadurch einen Beitrag zur besseren Beschreibung des menschlichen Denkprozesses.

\section{METHODE}

Bei der Untersuchung und Auswertung von Bildfolgen erschweren oftmals eine große Datenfülle und eine Vielzahl redundanter Bildinformation die Interpretation der Sequenzen. Eine effektive Analyse ist nur unter Ausnutzung von computergestützten Methoden der Bildverarbeitung, Datenreduktion und Datenkompression möglich. Der hier dargestellte Lösungsansatz wird diesen Anforderungen dadurch gerecht, daß er durch die Einbeziehung adaptiver rekursiver Schätzverfahren auf die Berechnung und Beobachtung von Bildparametern zu einer effektiven Verarbeitungsstruktur führt. Zur Applikation adaptiver rekursiver Schätzverfahren bieten sich zwei mögliche Richtungen an:

1. Schätzung von Bildparametern adaptiv rekursiv über die Bildfolge (z.B. Histogramm, Varianz, Quantil usw.)

2. Berechnung von Bildparametern für jedes Bild der Folge und Detektion von Instationaritäten im zeitlichen Verlauf des Parameters über adaptiv geschätzte Kenngrößen (z.B. Verlauf des globalen Kontrasts, darauf Anwendung einer adaptiven Quantilwertschätzung als Schwellwertdetektor).
In [1] wurden bereits Möglichkeiten vorgestellt. wie einfache adaptive rekursive Schätzverfahren (z.B. Mittelwert, 2.statistisches Moment, Quantilwert) in effektiver Art und Weise zu komplexen Schätzungen verknüpft werden können. Beispielhaft sei hier noch einmal die adaptive Varianz genannt, die sich als arithmetische Verknüpfung des 2.statistischen Moments und des adaptiven Mittelwertes ergibt. Eine breite Palette von Auswahlmöglichkeiten adaptiv gestalteter Schätzfunktionen gestattet das Setzen von Segmentgrenzen nicht nur auf der Basis visuell beobachtbarer Ähnlichkeiten von Teilfolgen in der Mapsequenz, sondern auch auf solchen, die über mathematische Kenngrößen definiert sind. So können z.B. Figuren den gleichen globalen Kontrast haben, obwohl sie visuell unähnlich sind. Auf dieser Grundlage wurden eine Vielzahl der aus der Bildverarbeitung zur Bildbeschreibung bekannten Parameter auf ihre Eignung zur Segmentierung untersucht. $\mathrm{Zu}$ nennen sind z.B. Grauwerthistogramm, CoOccurence-Matrix, globaler Kontrast, Entropie usw. Da eine einzelne Kenngröße zur Segmentierung aber ungeeignet scheint, wurde nach Verknüpfungsfunktionen mehrerer Kenngrößen bzw. nach weiteren Beschreibungsmöglichkeiten der Bildfolgen gesucht. Dabei erwiesen sich adaptive Schätzfunktionen in Matrix- oder Vektorform als aussagekräftige Parameter. So kann über

$$
\left.H_{x}=\left|M^{c}\right| I_{K_{1}}(x)\left|\ldots M^{c}\right| l_{K_{k}}(x)\right) \mid
$$

das Histogramm der Bildfolge adaptiv rekursiv im Zeitverlauf geschätzt werden, wobei Gl.(1) als Vektor von adaptiven Mittelwertschätzungen aufzufassen ist. Als Maß zur Einschätzung von Bildänderungen kann die Varianz über die Bildfolge angesehen werden. Eine effektive Umsetzung erhält man, indem man jedes Pixel getrennt in seinem Verlauf über die Bildfolge betrachtet. und die adaptive Varianz wie oben beschrieben über diese Zeitreihen ermittelt. So entsteht pro Pixel für jedes Bild der Bildsequenz ein Wert der Varianz, der wiederum in einem Bild visualisiert werden kann (siehe auch Abb. 2). Dabei entsprechen dunkle Farben einer kleinen Varianz, was bedeutet, daß bei einem sehr dunklen Bild der Varianz zwischen aufeinanderfolgenden Bildern der Bildfolge wenig Änderung stattgefunden hat. Auf dieser Grundlage lassen sich Segmentierungen in der Bildsequenz vornehmen.

\section{ERGEBNISSE}

Bei der Untersuchung kognitiver Prozesse hat sich die spektrale Kenngröße Kohärenz, und dabei speziell die lokale Kohärenz, als ein effektiver Parameter zur Beschreibung des funktionellen Zusammenhangs zwischen 
Gehirnarealen herausgestellt. Durch eine adaptiv rekursive Berechnungsvorschrift (u.a. in [4]) erhält man eine zeitlich und spektral hochaufgelöste Analysemethode, die zu jedem Meßpunkt auch eine Berechnung der Kohärenz erlaubt. Visualisiert man diese 30 Kanäle der lokalen Kohärenz in Mapsequenzen (Abb.l, pro Map 64*64 Pixel), so könnte man aufgrund visueller Ähnlichkeiten eine Einteilung in Segmente vomehmen. Anlehnend an die Segmentierungsverfahren von Lehmann und Strik ([2] und [3]) für

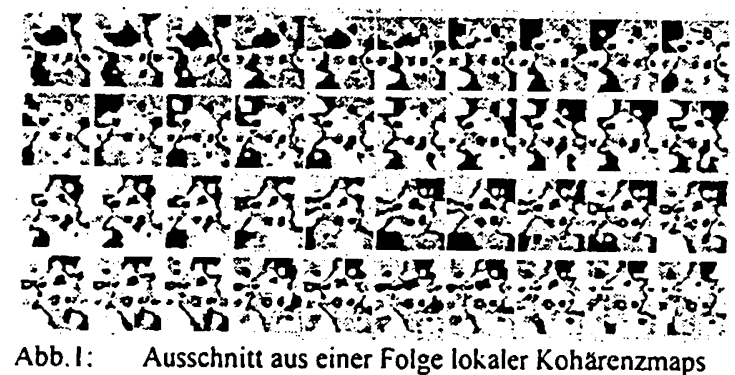

EEG-Potentialmapfolgen, wird folgender Lösungsweg auf der Grundlage von Bildinformationen vorgeschlagen.

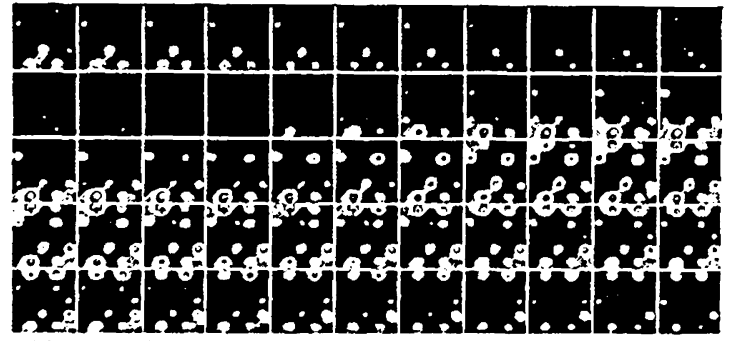

Abb.2: Folge komplexer adaptiver Varianzschatzungen

Die größte Änderung zwischen aufeinanderfolgenden Bildern liegt offensichtlich immer dann vor, wenn die Varianz maximal ist (Global Field Power bei Leh-

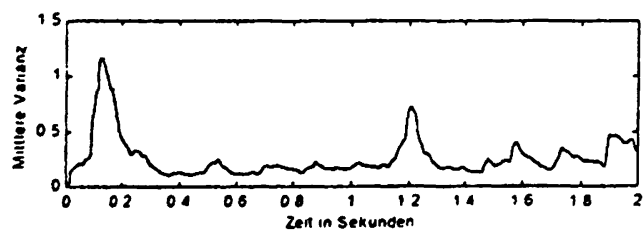

Abb.3: Zeitlicher Verlauf der mittleren Varianz über die Varianzmapfolge

mann/Strik). Dazu wird die Varianz über die Bildsequenz wie oben beschrieben berechnet und visualisiert (Abb.2). Ein Maß für die Gesamtvarianz pro Varianzmap kann z.B. die mittlere Varianz ubber das Map sein (Abb.3).

Immer wenn diese ein Maximum hat, ist die großte Bildănderung zu erwarten. In [2] und [3] werden zu diesen Zeitpunkten die Lageănderungen der Centroiden der Feldverteilung erfaßt. Dazu wird um die Centroidenposition ein kreisformiges Fenster gelegt, und zum năchsten Maximum der Global Field Power untersucht, ob die Centroidenposition das Fenster verlassen hat. In diesem Fall wird ein neues Segment eroffnet. Im vorliegenden Anwendungsfall wird der Schwerpunkt des Maps auf seine Lageănderung untersucht (Abb.4). Das zugrundeliegende

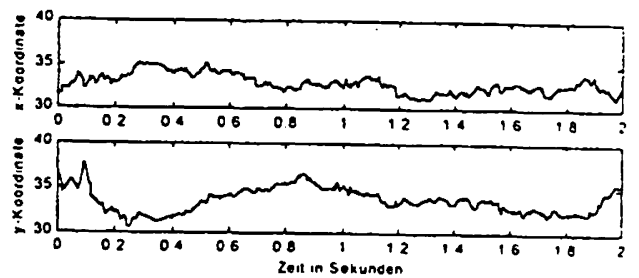

Abb.4: Zeitliche Koordinatenverlăufe des Mapschwerpunktes

Fenster wird variiert und nach der Zahl der so entstehenden Segmente analysiert (Abb.5). Ein sehr kleines Fenster führt zu vielen Segmenten, während ein großes Fenster nur wenige Segmente erzeugt. Unter Berücksichtigung physiologischer Bedingungen läßt sich nach [3] ein Optimum

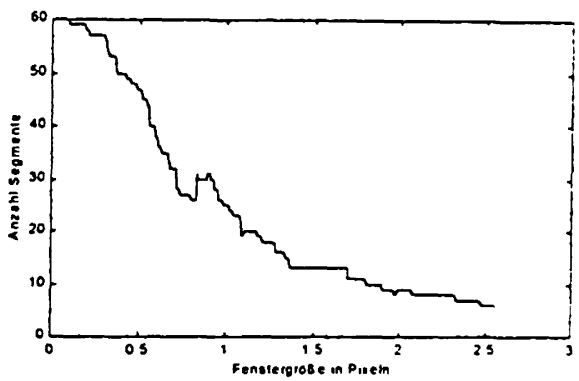

Abb.5: Anzahl der Segmente in Abhangigkeit der Fenstergroße

der Fenstergröße finden, was zu einer definierten Zahl von Segmenten führt. Diese Untersuchungen sind derzeit in Bearbeitung, ebenso wie ein Vergleich der Segmentierungsergebnisse mit einem Verfahren nach Schack. das in [4] vorgestellt wurde. Eine inhaltliche Interpretation durch einen Psychologen schließt sich an.

\section{ZUSAMMENFASSUNG}

Es konnte gezeigt werden, daß sich adaptive rekursive Schätzfunktionen sehr gut eignen, Instationaritäten in Bildfolgen und daraus abgeleiteten Parameterfolgen 2.1 detektieren und somit eine Segmentierung von Bildfolsectl zu ermöglichen. Weitere Untersuchungen müssen hluren. inwieweit zusätzliche Bildparameter eine Verbiscrun!: der Segmentierung bringen.

\section{LITERATUR}

[1] Steuer D.. Grießbach G. (1997): Komplei jJ.ını. Schătzalgorithmen in der Biosignalverarbeitung. Bi(m) sche Technik. Band 42. Ergänzungsband 2. S.21.22. 1M-1 0939.4990

[2] Lehmann, D.. Ozaki. H.. Pal. J. (1987a) lli (i . Inht.l map series: brain micro-states b! space-oriented addrul segmentation. Electroenceph. Clin. Neurophysiol. 67. 271 -2xis [3] Strik. W.K.. Lehmann. D. (1993) Data-decrmminil window size and space-oriented segmentation of spontansins EEG map series. Electroencephalography and clinical Ncuruph!. siology. 87. 169-174

[4] Schach. B. (1997) Adaptive Verliahren AII Sichtrol.

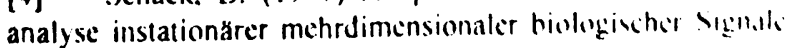
Habilitationsschrift. TU! Ilmenau

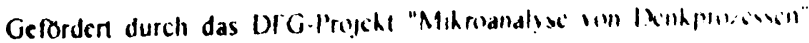
Nr. GRI\$\$s/I.1.

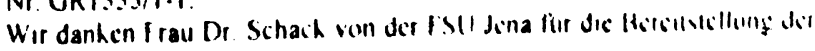
Daten und illerer Figchnisse 